Open Access

\title{
Influence of supplemental parenteral nutrition approach on nosocomial infection in pediatric intensive care unit of Emergency Department: a retrospective study
}

Dan Wang ${ }^{1}$, Xiaoquan Lai ${ }^{2}$, Chenxi Liu' ${ }^{1}$, Yuqi Xiong ${ }^{1}$ and Xinping Zhang ${ }^{1 *}$

\begin{abstract}
Background \& aims: Nutritional support for patients in the intensive-care unit (ICU) is a part of standard care which promotes medical quality and decreases nosocomial infection. Supplemental parenteral nutrition (SPN) approach (enteral nutrition (EN) combined with parenteral nutrition (PN) when EN alone is insufficient) has become one major concern in nutrition research field. This research aims to explore the following relationships: (i) the relationship between SPN and nosocomial infection, (ii) the relationship between early and late SPN initiation and the development of nosocomial infection.

Methods: A retrospective study was conducted in patients who met the inclusion criteria from February 2012 to February 2015 in Pediatric ICU (PICU). Patients were classified into two groups according to nutrition delivery approach-SPN group and EN alone group. Then SPN group were further divided into two subgroups by initiation timing, which were defined as early-initiation SPN and late-initiation SPN group respectively. Age, gender, serum albumin at admission, severity of disease, length of stay in PICU, nutrition delivery approach, amounts of delivered caloric intake and occurence of nosocomial infection were recorded. Univariate analysis and binary logistic regression analysis were performed to identify the risk factors and assess the independent effect of SPN approach on nosocomial infection in PICU of Emergency Department.
\end{abstract}

Results: 204 patients were included in our study. Compared with EN alone group, patients delivered by SPN approach had a higher nosocomial infection rate (34.0 vs.10.9\%, $p<0.001$ ). The late-initiation subgroup of SPN approach was found to be an independent predictor of nosocomial infection in the logistic regression analysis model $(\mathrm{OR}=3.40$; $95 \% \mathrm{Cl}, 1.13 \sim 10.19 ; p=0.029)$. Serum albumin at admission ( $\mathrm{OR}=0.91 ; 95 \% \mathrm{Cl}, 0.84 \sim 0.97 ; p=0.008)$, mechanical ventilation $(\mathrm{OR}=3.85 ; 95 \% \mathrm{Cl}, 1.43 \sim 10.39 ; p=0.008)$, severity of disease $(\mathrm{OR}=3.79 ; 95 \% \mathrm{Cl}, 1.03 \sim 13.99$; $p=0.045)$ and PICU length of stay $(\mathrm{OR}=1.23 ; 95 \% \mathrm{Cl}, 1.11 \sim 1.35 ; p<0.001)$ were also identified as significant risk factors for nosocomial infection.

Conclusions: Our study shows late-initiation SPN approach increases the incidence of nosocomial infection compared with early-initiation approach in critically ill children in PICU of Emergency Department. Compared with EN alone group, patients delivered by SPN approach had a higher nosocomial infection rate.

Keywords: Supplemental parenteral nutrition, Critically ill children, Nosocomial infection

\footnotetext{
* Correspondence: xpzhang602@163.com

${ }^{1}$ School of Medical Management and Health Management, Tongji Medical College of Huazhong University of Science and Technology, Wuhan, China Full list of author information is available at the end of the article
} 


\section{Background}

Nutritional support is vital for patients in the intensivecare unit (ICU). Despite increasing awareness of nutrition support, malnutrition is still prevalent in Pediatric ICU patients, with reports in the range of 25 to $45 \%$ at the time of admission [1-3]. Malnutrition has been identified as an independent factor for higher rate of nosocomial infections, which has become one of the most severe clinical outcomes associated with substantial morbidity and mortality, prolonged hospital stay and increased economic burden $[4,5]$. Therefore, it is of great significance to explore nutrition support for pediatric patients with nosocomial infection being the main focus of clinical outcome.

Driven by improving clinical outcomes and decreasing complex complications, nutrition delivery approach has been a major concern in nutrition research field for decades. Early enteral feeding approach has been preferred based on several guidelines [6-8]. In addition, substantial evidence showed enteral nutrition (EN) approach has been associated with improved clinical outcomes compared with parenteral nutrition (PN) approach $[9,10]$. However, critically ill patients often can not meet their energy target due to various situations and interruptions, such as medical investigations, surgery or gastrointestinal intolerance [11]. Therefore, PN approach was taken for critically ill patients. Although energy balances can be achieved by PN approach in short terms, most published studies showed PN approach resulted in increased infectious complications [12, 13]. Under this setting, researchers started to explore SPN approach (EN combined with PN when EN alone is insufficient) as one combination approach. But what are the benefits and potential harm of SPN approach compared to EN approach is still unknown when usual nutrition target is not met. And previous studies have put forth conflicting recommendations about SPN approach. The findings of Heidegger, Villet and Claude Pichard support potential value of SPN as one effective approach to reduce nosocomial infections [14-16], whereas some other studies suggest opposite conclusion and recommend that $\mathrm{PN}$ not be used combined with EN [17, 18].

Moreover, as timing of nutrition support being an important factor which would contribute to clinical outcomes, it was absent or poorly controlled in previous studies. Guidelines recommend early initiation of EN approach within $48 \mathrm{~h}$ at admission $[8,9,12]$. However, optimal timing for the initiation of PN still remains controversial [19]. Whether early or late-initiation of SPN approach influence the incidence of nosocomial infection is worth further exploring.

In this study, we aimed to explore two relationships under the current practice in PICU of Emergency Department: (i) the relationship between SPN and nosocomial infection, (ii) the relationship between early and late SPN initiation and the development of nosocomial infection.

\section{Methods \\ Study patients}

This retrospective study was conducted in PICU of Emergency Department which enrolled non-surgical patients in a tertiary hospital in Wuhan, China. The tertiary hospital covered over 4000 beds and reached 4280,000 outpatients and inpatients amounts in 2014. All patients aged younger than 18 years were enrolled on admission to the hospital PICU of Emergency Department for longer than $48 \mathrm{~h}$ and could tolerate some amount of enteral nutrition from February 2012 to February 2015. The patients who had more than one PICU admission during hospitalization, or had been referred to the PICU from other wards in the hospital and already received EN or PN, or were considered ineligible for EN by the physician due to contraindications of EN support during the PICU stay (including complete intestinal obstruction, necrotizing enterocolitis, gastrointestinal dysfunction caused by failure, severe infection, trauma and postoperative digestive tract paralysis and high-flow intestinal fistula) were excluded for this study. The study was approved by Ethics Committee of Tongji Medical College, Huazhong University of Science and Technology (IORG: IORG0003571).

\section{Data collection}

Patients' demographic and clinical characteristics were obtained from the Hospital Information System (HIS). These included sex, age, admittance date, serum albumin at admission, severity of disease, mechanical ventilation, invasive diagnosis operation and PICU length of stay. The nutritional data for the date that nutritional support was initiated, daily caloric intake and occurrence of nosocomial infection was also collected.

\section{Definition of nutritional support groups}

Two groups of patients were generated based on their nutrition delivery approach. The EN alone group included patients who only received EN while the other group received SPN support combined with EN during the PICU stay. To control the influence of timing, patients in SPN approach were further classified into two subgroups. Subgroup 1 included patients who initiated SPN in $48 \mathrm{~h}$ at admission, which was defined as earlyinitiation group. Subgroup 2 defined as late-initiation group included patients who initiated SPN between 2th day and 6th day of PICU stay. The physicians determined nutritional support for patients in PICU of Emergency department for most cases guided by standard operation procedure [20], and consultation of the pediatric nutritionist was initiated in complex cases only. 
If patients had functioning gastro-intestinal tract and were anticipating to remain unable to take oral nutrition for more than 3-7 days, EN would be given. Enteral calories were delivered by oral diet, nasogastric or nasojejunal tubes, and the feeding tubes were inserted by trained nurses. EN was delivered by feeding drip at a constant speed of $10-20 \mathrm{ml} / \mathrm{kg} / \mathrm{d}$. The volume of gastric residual was measured 4 to 6 times a day. If the clinician felt that the children could not achieve the target caloric intake (100 \% caloric requirement per day) by enteral feedings alone for more than 5 days, SPN would be delivered in our study. Parenteral calories were delivered in a mixed way by ready-to-mix 3-chamber bag (Kabiven G11 \%, Fresenius Kabi AB Sweden) or by the physicians' preparation containing amino acids, glucose, lipids, and electrolytes, and trace elements, minerals, and vitamins were added as clinically appropriate. We characterized patients as "enteral tolerant" if they received $\geq 1000$ enteral calories $/ \mathrm{kg} /$ day at any point within the PICU stay. The threshold for "enteal tolerant" was one arbitrary selection for the "enteral tolerant" intake requirements used by the attending physicians in the hospital. Predicted energy expenditure (PEE) calculated by the WHO equation [21] and daily caloric intake abstracted from the HIS was used for our analyses.

\section{Definition for some risk factors}

Age groups were classified by the criteria of American Centers for Disease Control and Prevention [22]. Severity of disease was scored into two levels by the judgment of physicians with a comprehensive evaluation of patient's condition, nursing and treatment plans and clinical emergency, such as coma or shock [23]. Serum albumin at admission was taken as one indicator for primary nutrition evaluation. Invasive diagnosis operation was defined as invasive examinations which concluded lumbar puncture, sternal puncture and abdominal puncture [24].

\section{Study outcomes}

The primary study outcome was the incidence of nosocomial infection. Infections were defined according to the definition from Chinese Diagnostic Criteria for Nosocomial Infection [25]. Five infection categories were defined: pneumonia(ventilator or non-ventilator-associated pneumonia, and other lower respiratory tract infections), bloodstream infection(laboratory-confirmed bloodstream infections and clinical sepsis); urogenital infection(device-associated or non-device associated urinary tract and genital infection); abdominal infection(intra-abdominal infections); and other infection [14].

\section{Statistical analysis}

Continuous variables such as age and weight were compared using a Students' T-test or Mann-Whitney U; chi-square and Fisher's exact test were used to compare categorical variables such as timing of PN initiation and sex. Univariate analysis was used to compare these factors for patients with and without nosocomial infection. Binary logistic regression was used to evaluate the independent impact of nutrition support approach and identify the independent impact of risk factors on nosocomial infection. Study variables which were significant at a 2-tailed $p<0.05$ in the univariate analysis or considered important were entered into the binary logistic model. Normality and homoscedasticity were checked previous to the implementation of parametric statistical tests. Variables which did not follow normal distribution were conducted for logarithmic transformation. Statistical analyses were performed with SPSS 12.0 (SPSS Inc., Chicago, IL, USA).

\section{Results}

During the 3-year study period 204 children were enrolled in our research. The flow diagram of patient recruitment was presented in Fig. 1. Characteristics of our study patients were listed in Table 1 . There were $65.7 \%$ male and $34.3 \%$ female, and patient received 287.7 $(179.3-411.3) \mathrm{kcal} /$ day. With the 46 nosocomial infection cases, pneumonia and bloodstream infection took the majority (56.52 and $34.78 \%$ for pneumonia and bloodstream infection respectively).

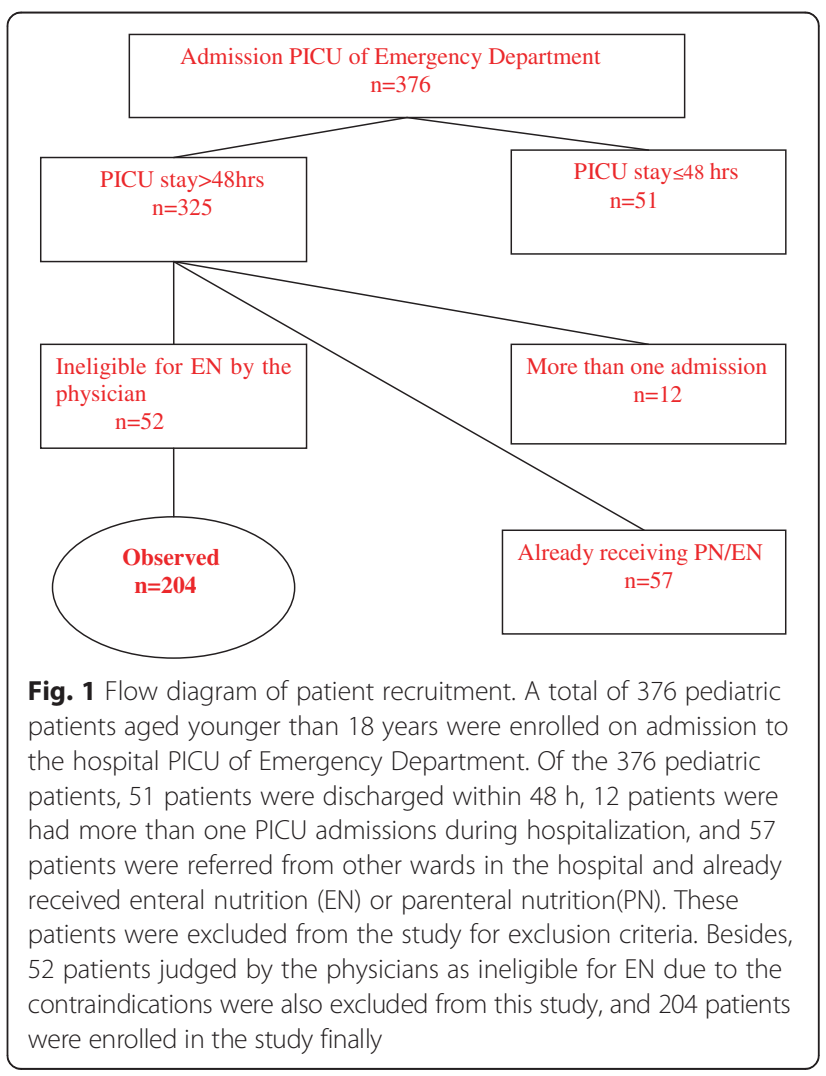


Table 1 Characteristics of study patients $(n=204)$

\begin{tabular}{lc}
\hline Characteristics & $n(\%) /$ median (IQR) \\
\hline Gender, $n$ (\%) & $134(65.7)$ \\
Male & $70(34.3)$ \\
Female & $9.0(3.0-30.0)$ \\
Age (month), median (IQR) & $8.1(5.0-13.0)$ \\
Weight (kg), median (IQR) & \\
Severity of disease, $n$ (\%) & $86(42.2)$ \\
Class 1 & $118(57.8)$ \\
Class 2 & $287.7(179.3-411.3)$ \\
Caloric intake (kcal/day), median (IQR) & $46(22.6)$ \\
Nosocomial infection, $n(\%)$ & \\
Type of nosocomial infection, $n$ (\%) & $26(56.5)$ \\
Pneumonia & $16(34.8)$ \\
Bloodstream infection & $3(6.5)$ \\
Urogenital infection & $1(2.2)$ \\
Abodominal infection & \\
Type of nutritional support, $n$ (\%) & $101(49.5)$ \\
EN alone group & $60(29.4)$ \\
Early-initiation group & $43(21.2)$ \\
Late-initiation group & \\
\hline
\end{tabular}

Note: IQR interquartile ranges (25th to 75 th percentile)

Of the whole 204 study patients, 101 (49.5\%) received EN alone and 103 (50.5 \%) received SPN. Their demographics, clinical characteristics, data for nutrition support and clinical outcome were presented in Table 2 . Patients receiving SPN showed a significantly higher percentage of nosocomial infection than patients receiving EN alone (34.0 vs. $10.9 \% ; p<0.001)$. All patients in EN alone group were initiated within $48 \mathrm{~h}$, while 58.3 and $41.7 \%$ patients in SPN group were initiated within $48 \mathrm{~h}$ and between 2 th day and 6th day respectively. For demographic factors, weight showed significant difference that children in SPN group were heavier than children in EN alone group. All clinical factors included showed significant difference in two groups. Patients in SPN group had lower level of serum albumin at admission, higher percentage of more severe condition (Class 2), mechanical ventilation, invasive diagnosis operation and longer PICU length of stay than those in EN alone group.

Patient characteristics of two subgroups of SPN approach were also presented in Table 2. Subgroup 2 had a higher percentage of nosocomial infection than subgroup 1 (46.5 vs.25.0 \%, $p=0.02$ ). Gender showed significant difference with more males in subgroup 1. Age, age groups, weight and clinical factors showed no significant difference in two subgroups.

Univariate relationship between independent predictors and occurrence of nosocomial infection were reported in Table 3. The incidence of nosocomial infection was significantly different in relation to nutrition delivery approach: EN alone approach $23.90 \%$, early-initiation approach $28.30 \%$ and late-initiation approach $47.80 \%$ $(p<0.05)$. Compared with patients with non-nosocomial infection, patients with nosocomial infection had significantly lower level of serum albumin at admission, more severe conditions(Class 2), higher rate of mechanical ventilation and invasive diagnosis operation and longer PICU length of stay.

To evaluate the independent effect of nutrition delivery approach on nosocomial infection, we conducted a binary logistic regression model. Variables included in initial regression were from study variable that were $<0.05$ in the univariate analysis or considered important. The results indicated that late-initiation SPN approach was associated with increased nosocomial infection. Serum albumin at admission, mechanical ventilation, severity of disease and PICU length of stay were also found as significant predictors of nosocomial infection (Table 3).

\section{Discussion}

In this study, 46 nosocomial infection cases were included. With only four cases of other types of nosocomial infection, the majority of nosocomial infecteions were distrubuted in pneumonia and bloodstream infection. It was likely due to the condition that PICU of Emergency Department in the hospital excluded the surgical patients. We also found that late-initiation SPN approach was associated with an increased risk of nosocomial infection compared with earlyinitiation SPN approach, while the association between early-initiation SPN approach and nosocomial infection was not demonstrated.

Our results were similar to one observational study conducted by Matthew J Sena in critically ill trauma patients. Their findings validated the adverse effect of SPN approach in critically ill patients [17]. It showed an increased association between early SPN and nosocomial infection. But early SPN was defined as SPN initiated within 1 week, which was slightly different from our study, in which $48 \mathrm{~h}$ was set as criteria. Additionally, our findings do support other studies as for other risk factors from the binary regression analysis. Serum albumin at admission, mechanical ventilation and severity of disease showed significant association with nosocomial infection, and these risk factors have been demonstrated by other studies [26-29]. PICU length of stay was also found as one predictor on nosocomial infection [30]. Although our results were similar to the previous studies, we still need to be cautious about interpreting our results, because previous studies did not take into account of timing factor or took different definition for the timing factor.

However, our findings violate results of several previous studies and guidelines which recommend SPN as one 
Table 2 Distribution of clinical outcomes, nutrition factors and other characteristics of patients

\begin{tabular}{|c|c|c|c|c|c|c|c|c|}
\hline Characteristics & EN alone group $(n=101)$ & SPN group $(n=103)$ & Statistic value & $P$ value & SPN subgroup1 $(n=60)$ & SPN subgroup2 $(n=43)$ & Statistic value & $P$ value \\
\hline Nosocomial infection, $n$ (\%) & $11(10.9)$ & $35(34.0)$ & $15.57^{\mathrm{a}}$ & $<0.001$ & $15(25.0)$ & $20(46.5)$ & $5.17^{a}$ & 0.02 \\
\hline Total caloric intake (\%), median (IQR) & $64.1(46.9-83.6)$ & $64.8(51.7-85.7)$ & $-0.29^{* b}$ & 0.77 & $65.42(50.8-82.9)$ & $64.80(52.4-86.1)$ & $0.57^{\mathrm{b}}$ & 0.57 \\
\hline Caloric intake (kcal/day), median (IQR) & $284.1(178.9-401.0)$ & $299.5(179.6-480.1)$ & $5163.00^{c}$ & 0.93 & $313.6(179.0-420.7)$ & $268.9(179.6-542.4)$ & $0.19^{\mathrm{b}}$ & 0.74 \\
\hline Gender, $n(\%)$ & & & $0.04^{\mathrm{a}}$ & 0.85 & & & $6.26^{\mathrm{a}}$ & 0.01 \\
\hline Male & $67(66.3)$ & $67(65.0)$ & & & $45(75.0)$ & $22(51.0)$ & & \\
\hline Female & $34(33.7)$ & $36(35.0)$ & & & $15(25.0)$ & $21(49.0)$ & & \\
\hline Age (month), median (IQR) & $9.0(3.0-26.0)$ & $9.0(3.0-37.0)$ & $3.44^{* b}$ & 0.07 & $11.0(3.0-49.0)$ & $7.0(4.0-30.0)$ & $0.17^{\mathrm{b} *}$ & 0.86 \\
\hline Age groups, $n(\%)$ & & & $2.34^{\mathrm{a}}$ & 0.50 & & & $5.54^{d}$ & 0.07 \\
\hline Infants & $58(57.4)$ & $55(53.4)$ & & & $31(51.7)$ & $24(55.8)$ & & \\
\hline Toddlers & $23(22.8)$ & $21(20.4)$ & & & $11(18.3)$ & $10(23.3)$ & & \\
\hline Preschoolers & $9(8.9)$ & $8(7.8)$ & & & $7(11.7)$ & $1(2.3)$ & & \\
\hline Childhood and young teens & $11(10.9)$ & 19 (18.4) & & & $11(18.3)$ & $8(18.6)$ & & \\
\hline Weight (kg), median (IQR) & $8.2(5.5-12.3)$ & $8(4.5-15.0)$ & $5.00^{* b}$ & 0.03 & $8.8(4.6-16.0)$ & $8.0(4.5-15.0)$ & $0.12^{\mathrm{b} *}$ & 0.91 \\
\hline Serum albumin at admission $(\mathrm{g} / \mathrm{L})$, mean $\pm \mathrm{SD}$ & $38.50 \pm 6.30$ & $35.94 \pm 6.11$ & $2.94^{\mathrm{b}}$ & 0.004 & $35.95 \pm 6.33$ & $35.94 \pm 5.86$ & $0.01^{b}$ & 0.99 \\
\hline Severity of disease, $n(\%)$ & & & $21.69^{\mathrm{a}}$ & $<0.001$ & & & $3.77^{\mathrm{a}}$ & 0.05 \\
\hline Class 1 & $59(58.4)$ & $27(26.2)$ & & & $20(33.3)$ & $7(16.3)$ & & \\
\hline Class 2 & $42(41.6)$ & $76(73.8)$ & & & $40(66.7)$ & $36(83.7)$ & & \\
\hline Mechanical ventilation, $n(\%)$ & $11(10.9)$ & $29(28.2)$ & $9.64^{\mathrm{a}}$ & 0.002 & $15(25.0)$ & $14(32.6)$ & $0.71^{\mathrm{a}}$ & 0.40 \\
\hline Invasive diagnosis operation, $n$ (\%) & $18(17.8)$ & $35(34.0)$ & $6.92^{\mathrm{a}}$ & 0.009 & $20(33.3)$ & $15(34.9)$ & $0.03^{\mathrm{a}}$ & 0.87 \\
\hline PICU length of stay (day), median (IQR) & $4.0(3.0-7.0)$ & $8.0(5.0-14.0)$ & $2922.00^{c}$ & $<0.001$ & $7.5(4.0-14.0)$ & $9.0(6.0-14.0)$ & $-0.53^{b}$ & 0.59 \\
\hline
\end{tabular}

Note: Variables with * were conducted for logarithmic transformation. Statistic values with ${ }^{\mathrm{a}, \mathrm{b}} \mathrm{c}, \mathrm{d}$ were Pearson's chi-square value, Students' $\mathrm{t}$ value and Mann-Whitney $\mathrm{U}$ value and Fisher exact test value respectively 
Table 3 Independent predictors for nosocomial infection

\begin{tabular}{|c|c|c|c|c|c|c|c|c|}
\hline \multirow{2}{*}{\multicolumn{2}{|c|}{ Characteristics }} & \multicolumn{4}{|l|}{ Univariate } & \multicolumn{3}{|c|}{$\underline{\text { Binary logistic regression }}$} \\
\hline & & $\begin{array}{l}\text { Non-nosocomial } \\
\text { infection }(n=158)\end{array}$ & $\begin{array}{l}\text { Nosocomial } \\
\text { infection }(n=46)\end{array}$ & Statistic value & $P$ value & $\overline{O R}$ & $95 \% \mathrm{Cl}$ & $P$ value \\
\hline \multicolumn{2}{|c|}{ Nutrition route, $n$ (\%) } & & & $22.80^{a}$ & $<0.05$ & & & \\
\hline \multicolumn{2}{|c|}{ EN only group } & $90(57.0)$ & $11(23.9)$ & & & & & 0.090 \\
\hline \multirow[t]{2}{*}{ SPN group: } & Subgroup1 & $42(26.5)$ & $13(28.3)$ & & & 3.40 & $1.13 \sim 10.19$ & 0.029 \\
\hline & Subgroup2 & $26(16.5)$ & $22(47.8)$ & & & 1.87 & $0.56 \sim 6.22$ & 0.281 \\
\hline \multicolumn{2}{|c|}{ Total caloric intake (\%), median (IQR) } & $65.0(48.6-84.0)$ & $63.5(50.9-82.8)$ & $0.02^{* b}$ & 0.98 & & & \\
\hline \multicolumn{2}{|c|}{ Caloric intake (kcal/day), median (IQR) } & $285.3(170.5-400.6)$ & $302.8(222.2-509.3)$ & $1.77^{* \mathrm{~b}}$ & 0.08 & & & \\
\hline \multicolumn{2}{|l|}{ Gender, $n(\%)$} & & & $1.78^{\mathrm{a}}$ & 0.18 & & & \\
\hline \multicolumn{2}{|l|}{ Male } & $100(63.3)$ & $34(73.9)$ & & & & & \\
\hline \multicolumn{2}{|l|}{ Female } & $58(36.7)$ & $12(26.1)$ & & & & & \\
\hline \multicolumn{2}{|c|}{ Age (month), median (IQR) } & $8.5(3.0-30.0)$ & $10.0(4.0-54.0)$ & $1.39^{* b}$ & 0.17 & & & \\
\hline \multicolumn{2}{|c|}{ Age groups, $n(\%)$} & & & $2.45^{\mathrm{d}}$ & 0.48 & & & \\
\hline \multicolumn{2}{|l|}{ Infants } & $89(56.30)$ & $24(52.20)$ & & & & & 0.495 \\
\hline \multicolumn{2}{|l|}{ Toddlers } & $35(22.20)$ & $9(19.60)$ & & & 0.87 & $0.27 \sim 2.76$ & 0.809 \\
\hline \multicolumn{2}{|l|}{ Preschoolers } & $14(8.90)$ & $3(6.50)$ & & & 0.61 & $0.09 \sim 4.19$ & 0.602 \\
\hline \multicolumn{2}{|c|}{ Childhood and young teens } & $20(12.70)$ & $10(21.70)$ & & & 2.33 & $0.64 \sim 8.47$ & 0.198 \\
\hline \multicolumn{2}{|c|}{ Weight (kg), median (IQR) } & $8.0(5.0-12.6)$ & $9.0(6.0-17.5)$ & $1.61^{* b}$ & 0.11 & & & \\
\hline \multicolumn{2}{|c|}{ Serum albumin at admission $(\mathrm{g} / \mathrm{L})$, median $\pm \mathrm{SD}$} & $38.19 \pm 6.05$ & $33.85 \pm 6.16$ & $4.26^{\mathrm{b}}$ & $<0.001$ & 0.91 & $0.84 \sim 0.97$ & 0.008 \\
\hline \multicolumn{2}{|c|}{ Severity of disease, $n(\%)$} & & & $27.27^{\mathrm{a}}$ & $<0.05$ & 3.79 & $1.03 \sim 13.99$ & 0.045 \\
\hline \multicolumn{2}{|l|}{ Class 1} & $82(51.90)$ & $4(8.70)$ & & & & & \\
\hline \multicolumn{2}{|l|}{ Class 2} & $76(48.10)$ & $42(91.30)$ & & & & & \\
\hline \multicolumn{2}{|c|}{ Mechanical ventilation, $n$ (\%) } & $20(12.70)$ & $20(43.50)$ & $21.47^{\mathrm{a}}$ & $<0.05$ & 3.85 & $1.43 \sim 10.39$ & 0.008 \\
\hline \multicolumn{2}{|c|}{ Invasive diagnosis operation, $n(\%)$} & $30(19.00)$ & $23(50.00)$ & $17.82^{\mathrm{a}}$ & $<0.05$ & 1.49 & $0.55 \sim 4.07$ & 0.436 \\
\hline \multicolumn{2}{|c|}{ PICU length of stay (day), median (IQR) } & $5.0(3.0-8.0)$ & $12.5(8.0-15.3)$ & $1172.5^{c}$ & $<0.001$ & 1.23 & $1.11 \sim 1.35$ & $<0.001$ \\
\hline
\end{tabular}

effective way to reduce nosocomial infections [14-16, 31]. The possible factors for such disagreement are discussed as follows. Firstly, discrepancy in sample population may contribute to such difference. Sample population in our study was medical patients aged younger than 18 years while adult patients were chosen as study population in aforementioned studies. Children patients, as one group with specific physiological characteristics, were likely more sensitive to outside environment, such as intravenous -delivered route by SPN approach. Although studies which came to the same findings were also conducted in adult patients, children patients can not be ignored as one possible predictor for nosocomial infections. Based on such consideration, the variable of age groups which classified pediatric patients into four groups were analysed. However, the age groups showed no significant difference in both univariate and regression analyses. Considering the sample size and exclusion of adult patients in our study, future comparative studies can be done for exploring the difference between study patients. Another factor for our observation includes difference in caloric quantity. Previous studies showed different caloric quantities delivered by EN or SPN approach, in which one multi-center random controlled research designed combined feeding strategy with exact $100 \%$ provision of energy target. It is still not certain exactly how an increased number of calories might influence infection rate, regardless of the composition of nutrition support.

Based on foregoing discussion, one most possible explanation for association between late-initiation SPN approach and nosocomial infection in our study is PN approach itself. The common finding is PN route is associated with an increase in postoperative complications. Such finding has been demonstrated by other studies previously [32, 33]. Moreover, SPN group and EN alone group received similar proportion of their target requirements in our study in which controlled the influence of nutrition amount $(p>0.05)$, which controlled the effect of nutrition amount on nosocomial infection. Therefore, 
increased risk of nosocomial infection by SPN approach is likely, at least in part, due to PN approach itself. Although some available guidelines recommend $\mathrm{PN}$ when caloric goals are not met during the first week, it has not been shown that this approach is beneficial. A multicenter observational study conducted in 2011 showed the most common reason for initiation of PN was "No reason" [34]. The anxiety of providing no nutrition support possibly impacted the choice of the delivery of PN which leaded most clinicians to select SPN, and it still plays an important role under current nutrition practice. One more possible explanation is timing of SPN initiation. Timing is one potential factor which is still under debate for PN initiation. Patients in early-initiation and lateinitiation group showed different statistical results in binary logistic regression. Late-initiation SPN approach was likely associated with delayed energy balance, and negative energy balance has been validated to correlate with increased infections [15]. Under the circumstances of Emergency Department in our study, the need for quicker restoration of energy balance and important indices was likely more urgent, which may increase the risk for nosocomial infection.

There were several limitations in this study. Firstly, it was a retrospective study with a relatively small group of both the study population $(n=204)$ and the number of events $(n=46)$ in a single center, and this study is limited by the possibility that patients from SPN group and EN alone group differed systematically and the small size of events may preclude the observed difference to be significantly different. Thus the conclusion should be interpreted and applied with caution. Additionally, the study population only covered Chinese pediatric patients, which may limit the generalizability to other ethnic groups. Future studies should include a larger sample size and multiple institutions containing other ethnic groups. Secondly, our study was also limited due to some poorly defined concepts. Such concepts such as "adequate" or "sufficient" nutrition support and the criteria for "enteral tolerant", "early" or "late" initiation remained unclear in existing literatures and guidelines. It is hard to reach the consensus about whether $48 \mathrm{~h}$ or 1 week maybe better set as criteria for "early" initiation. We define "early" initiation of SPN approach as within $48 \mathrm{~h}$ at admission, referring to early initiation of EN approach and the nutrition practice in our study institution, and the threshold for "enteral tolerant" was an arbitrary selection which was defined as receiving $\geq 1000$ enteral calories $/ \mathrm{kg} /$ day at any point during PICU stay. Our study was also limited due to a lack of data on nutritional assessment measures, such as Nutritional Risk Index (NRI) score, and Body Mass Index (BMI) was also not available for the missing data of height for majority of pediatric patients. Thus we took serum albumin at admission as the alternate indicator for primary nutrition evaluation. And further random controlled studies need to be designed for controlling potential confounders, such as nutritional practices.

Even though our study was limited in some aspects as one retrospective study, our findings provided the new insights with regards to controversial nutrition topic concerning SPN approach and its delivery timing. Further researches would be encouraged to further explore and validate the SPN approach and its other aspects.

\section{Conclusion}

This study shows late-initiation SPN approach increases the risk of nosocomial infection compared with EN alone approach in PICU of Emergency Department. Serum albumin at admission, mechanical ventilation, severity of disease and PICU length of stay are important risk factors for nosocomial infections.

\section{Abbreviations}

PICU: Pediatric intensive care unit; EN: Enteral nutrition; PN: Parenteral nutrition; SPN: Supplemental parenteral nutrition; HIS: Hospital information system; NRI: Nutritional risk index; BMI: Body mass index.

\section{Competing interest}

The authors declare no conflict of interest or financial interest related to this research. The fund source provided financial support without other involvement in this research.

\section{Authors' contributions}

DW helped design the study, performed the data collection and initial analyses and drafted the initial manuscript. XL helped design the study, reviewed and revised the manuscript. $C L, Y X$, and $X Z$ helped reviewed and revised the manuscript. All authors read and approved the final manuscript as submitted.

\section{Acknowledgements}

This research was supported by a project named "The benchmark study of indicators measurement of health-care associated infection based on transparency" (Grant No. 71473098) from National Natural Science Foundation of China.

\section{Author details}

${ }^{1}$ School of Medical Management and Health Management, Tongji Medical College of Huazhong University of Science and Technology, Wuhan, China. ${ }^{2}$ Tongji Hospital, Tongji Medical College of Huazhong University of Science and Technology, Wuhan, China.

Received: 1 June 2015 Accepted: 26 September 2015

Published online: 06 October 2015

\section{References}

1. Hulst J, Joosten K, Zimmermann L, Hop W, van Buuren S, Buller $\mathrm{H}_{\text {, et al. }}$ Malnutrition in critically ill children: from admission to 6 months after discharge. Clin Nutr. 2004;23(2):223-32.

2. Menezes FS, Leite HP, Nogueira PC. Malnutrition as an independent predictor of clinical outcome in critically ill children. Nutrition. 2012;28(3):267-70.

3. Chong CY. Prevention of nosocomial bacterial infections in pediatrics and neonatology. J Microbiol Immunol. 2015;48 Suppl 1:S12.

4. Jones KDJ, Berkley JA. Severe acute malnutrition and infection. Paediatr Int Child Health. 2014:34(Suppl1):1-29.

5. Correia MITD, Waitzberg DL. The impact of malnutrition on morbidity mortality, length of hospital stay and costs evaluated through a multivariate model analysis. Clin Nutr. 2003;22:235-9.

6. Singer P, Berger MM, Van den Berghe $G$, et al. ESPEN Guidelines on Parenteral Nutrition: intensive care. Clin Nutr. 2009;28:387-400. 
7. Martindale RG, McClave SA, Vanek WW, McCarthy M, Roberts P, Taylor B, et al. Guidelines for the provision and assessment of nutrition support therapy in the adult critically ill patient: Society of Critical Care Medicine and American Society for Parenteral and Enteral Nutrition: Executive Summary. Crit Care Med. 2009;37:1757-61.

8. Heyland DK, Dhaliwal R, Drover JW, Gramlich L, Dodek P. Canadian clinical practice guidelines for nutrition support in mechanically ventilated, critically ill adult patients. J Parenter Enteral Nutr. 2003:27:355-73.

9. Simpson F, Doig GS. Parenteral vs. enteral nutrition in the critically ill patient: a meta-analysis of trials using the intention to treat principle. Intens Care Med. 2005;31:12-23.

10. Chung CK, Whitney R, Thompson CM, Pham TN, Maier RV, O'Keefe GE. Expereince with an Enteral-Based Nutritional Support Regimen in Critically II Trauma Patients. J Am Coll Surg. 2013;217:1108-17.

11. Hadfield RJ, Sinclair DG, Houldsworth PE, Evans TW. Effects of enteral and parenteral nutrition on gut mucosal permeability in the critically ill. Am J Respir Crit Care Med. 1995;152:1545-8.

12. Gramlich L, Kichian K, Pinilla J, Rodych NJ, Dhaliwal R, Heyland DK. Does enteral nutrition compared to parenteral nutrition result in better outcomes in critically ill adult patients? A systematic review of the literature. Nutrition. 2004;20:843-8

13. Moore FA, Feliciano DV, Andrassy RJ, McArdle AH, Booth FV, MorgensteinWagner TB, et al. Early enteral feeding, compared with parenteral, reduces postoperative septic complications: The results of a meta-analysis. Ann Surg. 1992;216:172-83.

14. Heidegger CP, Berger MM, Graf S, Zingg W, Darmon P, Costanza MC, et al. Optimisation of energy provision with supplemental parenteral nutrition in critically ill patients: a randomised controlled clinical trial. Lancet. 2013;381:385-93.

15. Villet S, Chiolero RL, Bollmann MD, Revelly JP, Cayeux RNMC, Delarue J, et al. Negative impact of hypocaloric feeding and energy balance on clinical outcome in ICU patients. Clin Nutr. 2005;24:502-9.

16. Pichard C, Thibault R, Heidegger C-P, Genton L. Enteral and parenteral nutrition for critically ill patients: A logical combination to optimize nutritional support. Clin Nutr. 2009;Suppl 4:3-7.

17. Sena MJ, Utter GH, Cuschieri J, Maier RV, Tompkins RG, Harbrecht BG, et al. Early supplemental parenteral nutrition is associated with increased infectious complications in critically ill trauma patients. J Am Coll Surg. 2008;207:459-67.

18. Heyland DK, Schroter-Noppe D, Drover JW, Jain M, Keefe L, Dhaliwal R, et al. Nutrition support in the critical care setting: current practice in Canadian ICUs_opportunities for improvement? JPEN J Parenter Enteral Nutr. 2003:27:74-83.

19. de Aguilar-Nascimento JE, Bicudo-Salomao A, Portari-Filho PE. Optimal timing for the initiation of enteral and parenteral nutrition in critical medical and surgical conditions. Nutrition. 2012;28:840-3.

20. Pediatric Collaborative Group, Society of Parenteral and Enteral Nutrition, Chinese Medical Association. Clinical guidelines for the provision of enteral nutrition and parenteral nutrition for children patients (in Chinese). Chinese Journal of Pediatrics. 2010:48:436-41.

21. World Health Organization. Energy and protein requirements. Report of a joint FAO/WHO/UNU expert consultation. WHO technical report series 724 . Geneva: World Health Organization; 1985.

22. American Centers for Disease Control and Prevention: child development. http://www.cdc.gov/ncbddd/childdevelopment. Accessed 19 Aug 2015.

23. Chinese Ministry of Health. Regulation of Nosocomial Infection Surveillance WS/T312-2009. Zhonghua Yi Yuan Gan Ran Xue Za Zhi. 2009;11:314-20.

24. Ouyang Q, Wu H, Liu C. Clinical Diagnostics (in Chinese). People's Medical Publishing House. 2nd ed. 2010

25. Chinese Ministry of Health. Diagnostic Criteria for Nosocomial Infection(tentative standard). National Medical Journal of China (in Chinese). 2001;81:314-20.

26. Llop JM, Muñoz C, Badía MB, Virgili N, Tubau M, Ramón JM, et al. Serum albumin as indicator of clinical evolution in patients on parenteral nutrition. Multivariate study. Clin Nutr. 2001;20:77-81.

27. Solomkin JS. Ventilator-associated pulmonary infection: the germ theory of disease remains viable. Microbes Infect. 2005:7:279-91.

28. Mireya UA, Martí PO, Xavier KV, Cristina LO, Miguel MM, Magda CM. Nosocomial infections in paediatric and neonatal intensive care units. J Infection. 2007:54:212-20.

29. Serón-Arbeloa C, Puzo-Foncillas J, Garcés-Gimenez T, Escós-Orta J, LabartaMonzón L, Lander-Azcona A. A retrospective study about the influence of early nutritional support on mortality and nosocomial infection in the critical care setting. Clin Nutr. 2011;30:346-50.

30. González-Cortés R, López-Herce-Cid J, García-Figueruelo A, Tesorero-Carcedo G, Botrán-Prieto M, Carrillo-Álvarez A. Prolonged stay in pediatric intensive care units: mortality and healthcare resource consumption. Med Intensiva. 2011;35:417-23.

31. Kreymann KG, Berger MM, Deutz NE, Hiesmayr M, Jolliet P, Kazandjiev G, et al. ESPEN guidelines on enteral nutrition: intensive care. Clin Nutr. 2006;25:210-23.

32. Rhee P, Hadjizacharia P, Trankiem C, Chan L, Salim A, Brown C, et al. What happened to total parenteral nutrition? The disappearance of its use in a trauma intensive care unit. J Trauma. 2007;63:1215-22.

33. Dhaliwal $R$, Jurewitsch B, Harrietha D, Heyland DK. Combination enteral and parenteral nutrition in critically ill patients: harmful or beneficial? A systematic review of the evidence. Intens Care Med. 2004;30:1666-71.

34. Kutsogiannis J, Alberda C, Gramlich L, Cahill NE, Wang M, Day AG, et al. Early use of supplemental parenteral nutrition in critically ill patients: results of an international multicenter observational study. Crit Care Med. 2011;39:2691-9.

\section{Submit your next manuscript to BioMed Central and take full advantage of:}

- Convenient online submission

- Thorough peer review

- No space constraints or color figure charges

- Immediate publication on acceptance

- Inclusion in PubMed, CAS, Scopus and Google Scholar

- Research which is freely available for redistribution 